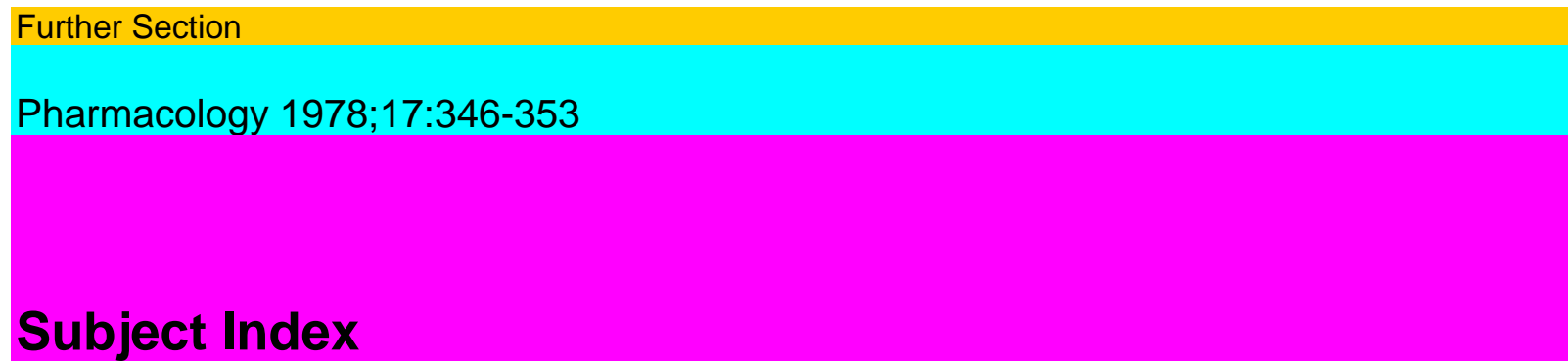

\title{
Absorption 330
}

- of fluids 332

-, variable lipid partition $(\mathrm{K})$ values

330 Abstinence, morphine 88 -, naloxone-induced 96 Acacia-gum solution 122 Accommodation 249 Acenocoumarin 233 Acetaldehyde 5 Acetanilide 191

- $\quad$-4-hydroxylase 192

Acetone 264

Acetylcholine 8, 83, Ill, 176, 210 -, activation of guanylate cyclase

280 Acetylsalicylic acid 79 -, fecal blood loss 81 -, ulcerogenic effect 79 Acid phosphatase 59

secretion 32, 254 Activation, lymphocyte 288 Adenyl cyclase 96, 128

-, dopamine-sensitive 280 Adjuvant arthritis 288, 328 /3-adrenergic blocking agents 323

Adrenergic receptors, alpha and beta

144 Agents, capable of antagonizing

SRS-A 323 -, ulcerogenic 254 Akinesia 211 Albumin, binding of coumarin

anticoagulants 234

- $\quad$-coumarin complexes,

spectroscopic data 236

Alcohol 158

Aldrin, epoxidation 201

Allergic asthma, human 323

- reactions 158

Allopurinol 116

7-amino-butyric acid 150, 210

6-aminodopamine 8

2-aminoethyl-2,2-diphenylpropyl-

acetate 208

Aminoglutethimide 264

Amino nitrogen, liver perfusate 184

Aminophenazone 307

5-amino-l-phenyltetrazole 56

Aminophylline 323

Aminopyrine 328

-, 14C 331

-, demethylation activity 169

Aminotriazole 21

Amphetamine 116, 158, 210, 227

Analgesia 96, 128, 215

Anaphylactic reactions 286

Anaphylaxis 323 
Angina pectoris 301

Animals, tumour bearing 295

Anorectic drugs,

phenylpropanolamine 157 Antagonism of SRS-A 323 Antianxiety agents 105 Antiarrhythmics

301 Antiasthmatic properties 323

- potential, oxarbazole 328

Anticholinergic action,

phenothiazines 285 Anticholinergics 104 Anticoagulants 233 Antidiabetic drugs 157 Anti-

inflammatory activity 56 Antiproteolytic activity 56 Antipyrine 201 -, '4C 331

-, HSLC chromatogram 101 - -N-,4CH3 98, 201 -, pharmacokinetic parameters 3 -, plasma 98 -, plasma half-life 201 Antithyroid drugs 28 Antitumor agents 61,116 Antiulcer activity, THC 176

Aorta 315 Aortic microsomes, preparation 3

- $\quad$ strips 315

Apomorphine 139

Appetite suppression 157

1-arabinose 45

Aromatic amino acid decarboxylase

149,153 Arterial blood pressure, effect of

strangulation 17 Arthritic rat serum, chromatography

194 Arthritis, adjuvant 288 Arylhydrocarbon hydroxylase 163,

192, 241 Arylsulfatase 102, 202 5-aryl-2-tetrazolylalkanoic acid 56 Asbestos granuloma, rats

328 Ascorbate 341

Aspartate aminotransferase 70 Aspirin 81, 328 Asthma 323 -, allergen-induced 328 Atropine

$110,325-, 3 \mathrm{H} 331$

Axon membrane potential 249 Azidomorphine 215

Baboon 221

Bacterial endotoxin 181

Barbital 335

-, 14C 331

Barbiturates 116

Barium, contraction of the ileum

longitudinal muscle 320 Basal ganglia, dissection 150 Behavior, stereotyped 211 Benzo(a)pyrene

191, 241 Benzodiazepine 104, 121 Benzpyrene, metabolites 243

hydroxylation 169 Benzyl alcohol 263

-amine 1, 116, 263

penicillin 339

-, 14C 331

methylamine 115

-/3-D-xyloside 52 Biguanides 158 Bile acid 69

formation, canalicular 69 Biliary tract spasms 230

Subject Index

347

Biogenic amines 8

Biological rhythms 88

Bio transformation 113, 163 
2-biphenylhydroxylation 39

Bisquarternary salts 61

/3-blockade 301

Blockade, histamine $\mathrm{H}_{1}^{-}$- or $\mathrm{H} 2$ -

receptor 37 Blood-brain barrier 110, 221

glucose 21, 157, 224

pressure 19

sugar utilization 157 Body temperature 29

weight $21,24,227$

_, in methadone-treated rats

227

- $\quad$ - loss 91

Bradykinin 323

Brain 8

-, concentrations of morphine 91

-, cyclic AMP 128

-, deficit of dopamine 210

- $\quad$ stem 1

Bronchoconstriction 323 BSP, bile flow and clearance 73 Buffering ability of the peritoneal cavity 333 Butanol 262

Caffeine, 3H 331 Calcium, antagonists 316 -, different stores 315,320 -, movements in microsomes 315 -, uptake and release 315 Calve 323 cAMP 21, 96 -, brain function 128 -, exogenous administration $135-, 3 \mathrm{H}$, formation and effect of morphine in CNS 131

- - phosphodiesterase, increase in

the high affinity 29

Canalicular bile formation 74 Cancer 295

- $\quad$ chemotherapy 331

Carbachol 323

Carbidopa 142

-, decarboxylase inhibitor 138 -, effect of pretreatment on the plasma growth hormone 142

Carbon disulfide 264

- $\quad$ tetrachloride 264

Carboxymethyl-cellulose 39, 289

Carcinogen 116

—, benzo(a)pyrene 191 Carcinogenesis 241 Carcinogenicity 191 Cardiac arrhythmias 301 output 305 Cardiotoxic peptide 188 Carrageenin 56

edema 57,328 Cartilage 50

Cat 15,182

-, isolated nictitating membrane

215 - , perfused liver 181 Catalepsy, morphine-induced 129 Catecholamines 29, 221 —, ability to alter pancreatic

hormone release 224 -, responsiveness 21

- $\quad$ sensitive adenylate cyclase,

supersensitjvity 285

-, urinary output 30 Catecholaminergic neurons, specific

destruction of 8 Cathepsin D 181 
D activities, in liver tissue 185

Caudiverbera sciatic nerve 250

Cells, lymph node 288

Centalun 116

Central action, morphine 128

activity 121 Cerebellum 285 Cerebral cortex 1, 128

hemispheres 9

- ischemia 15

Cerebrum 285

Cervical constriction 17

Chemical carcinogens, sensitivity

191 Chemotherapy, peritoneal 330 Children, born to methadone-treated

mothers 227 Chlordesmethyldiazepam 126 2'-chlor-D-desmethyldiazepam 121

Chlordiazepoxide 104 Chloroform 264 Chlorpromazine 70, 280

Chlorprothixene 69

-, effects on bile flow 72

Cholestatis 68

Cholesterol, dietary 163

Choline 83

Cholinergic-blocking agents 323

- stimulation 260

Chromatography 113, 241

Chronic administration of

methadone 227

- $\quad$ intake of levodopa 149

- methadone, effect on body

weight, food and water

consumption in rats 227

- $\quad$ renal failure 307

Cigarette smoking 192, 243

Circular dichroism 233

Circulatory shock 187

Clearance, 14c-erythritol 69

-, morphine 88

Clidinum bromide 104

Clozapine 211

CNS 135, 157

Cocaine 216

Collagen 50

—, incorporation of $7 / 8$ proline 53

Cold air 29

Colloidal carbon, half-time values for

clearance 186 Compounds, administered

intraperitoneally 330 -, lipid-soluble or unionized 330 -, neurotoxic 8 Concanavalin A 288

Conflict behavior 121

- $\quad$-, effects of desmethyldiazepam 
and chlordesmethyldiazepam 123

Conjugative enzymes, hepatic 69

Connective tissues 50

Continuous avoidance, effects of desmethyldiazepam and chlordesmethyldiazepam 124

Convulsion 211

Cortical slices 128

Cortisol 138

Contraction, calcium pool 316

-, norepinephrine induced 315

Corpus striatum 9

Corticosteroids 56

348

Subject Index

Coumachlor 233 Coumarin 197, 233

- $\quad$ anticoagulants, extrinsic cotton

235

Culture, arthritic rat lymphocytes

284 Cyclic AMP 128,280 Cyclic GMP 280 -, pulmonary tissue 285 Cyclohexane oxide 264 Cytidine diphosphocholine 15 Cytidoline 15

Cytochrome c reductase 163, 342 Cytochrome P-450 113, 163, 198, 262,342

- $\quad$ P,-450 (P-448) 246

Decongestant 157 Deficiency, vitamin B6 Degranulation, mast cell 36 Dehydrogenase 181

Dependence, morphine 88 Desmethyldiazepam 121 Dexamethasone 182 Dextran 338

- blue 333

Diabetes 157

Dialysis, peritoneal 330

Diarrhea 91

Diazepam 104, 121

Dichloromethane 193

Diencephalon 9

Dietary lipids, effect on microsomal

drug-metabolizing enzymes 168 Diet, cholesterol-free and rich 163 -, effect of different cholesterol

contents 165 -, ofpurinachow $150 / 3$-diethylaminoethyl

diphenylpropylacetate, SKF

525-A 201

- $\quad$ spectrophotometry 263

Difference spectra, effects of various

compounds in liver microsomes

268 Dihydroergotoxin 15 1-dihydroxyphenylalanine 138, 210, Dihydroxytryptamine 8

Dimethylsulfoxide 62 Disease, Parkinson 210

Disulfiram 116, 158 DNA 61

-, benzo(a)pyrene metabolites

bound to 241 Dog 138 
Dopa decarboxylase inhibitor 149 1-dopa, mechanism of hyperglycemia

138 Dopamine $1,8,83,86,138,149$,

210 -, determination 151

- $\quad$ hydroxylase 144

—, sensitive adenylate cyclase 280 Drug absorption, effect of dissociation constant 332

- _, lipid-water partition

coefficient effect 332

- $\quad$-, molecular weight effect 332

-, anorectic 157

-, anticholinergic 173 -, anti-inflammatory 328

binding 263

bio transformation 231

combination, quinitidine and propranolol 305

interactions 301

metabolism, dietary regulation 164

- $\quad$-metabolizing enzyme 163, 262

-, neurotoxic 13

-, non-steroidal anti-inflammatory

288 -, producing jaundice 70

- $\quad$ transport, stomach and small

intestine 330

Duodenal ulcer 104

Edema 56

-, nystatin 289

EEG 121

-, effects of desmethyldiazepam and

chlordesmethyldiazepam on mean

amplitude 125 Electrocardiography, after

quinitidine and during

propranolol 304 Electron transfer activities 39 Electrons, lonepair 271 Electrophoresis, agarose gel 295 Elimination 301

Emotional disease 109 Encephalomyelitis, allergic 197 Endoplasmic reticulum 39, 163, 186

Endotoxin 76, 181 -, effect on perfusate cathepsin 183 Enzymatic biotransformation, drug

and carcinogen 197 Enzyme assay 48 - , lysosomal 60 Epinephrine 29, 138, 143, 280 -,

hyperglycemic activities 221 Epoxidation 242

- $\quad$ high and low spin P-450 ferric

iron in vivo 273

Equilibrium, dialysis 308

Ergot alkaloids 20

Ergotamine 15

Erosions, gastric mucosal 104

-, ruminal 258

Erythrocytes, 51Cr-labelled 79

Erythromycin estolate 70

Escherichia coli 182 
Ethanol 1, 249

-, effect on action potentials of the

sciatic nerve 250 -, effect on MAO 3 Ethoxycoumarin 197

- deethylase 163

Ethyl acetate 264

2-ethylaminoethyl-2,2-diphenyl-

propylacetate 208 Ethylmorphine N-demethylase 113 Ethyl-(5-phenyltetrazol-2-yl)acetate 57

Ethynylestradiol 76 Experimental acute renal failure 307 Extrapyramidal system 210 Extrinsic cotton effects 233

Fate of drugs 307 Fecal blood loss 79 Feces 61 Feeding 157

- of antioxidants 341

Females, methadone-treated 227

Fenfluramine 158

Fetal brain 227

calf cartilage, organ culture 50

mortality 228 Ficoll 2

First-pass effect, phenacetin 195

Subject Index

349

Fluid retention 158 Follicular cell height 27 Food additives 39

- consumption, during gestation

and lactation 227

- $\quad$ intake 21

Forced exertion 104

Forepaw lifting 211

Formaldehyde 114

Formation, 3H-cAMP 128

Free fatty acids 29, 140

Frog sciatic nerve 249

1 -fucose 45

Fusaric acid 144

d-galactose 45 d-galacturonic acid 45 Gallamine 15

Gas liquid chromatography 98 Gastric acid neutralisation 258

- secretion, basal levels Ill

glandular lesions, incidence 36

HC1 32

lesions 33

mast cells 37,254

perfusion 32

secretion 254

ulcer 104, 254 Gastrin Ill Gastrointestinal hormones Ill

toxicity 79 Geller'stest 124 Gestation 227

Glandular gastric mucosa 256

globulins 288 Glucagon 29, 221 Glucosaminoglycans 50 Glucose 29

metabolism 138 
-6-phosphate dehydrogenase 114

uptake 138

utilization 139 Glucosuria $161 \alpha$-glucuronic acid 45 ß-glucuronidase 59, 202 Glutamic acid 150

Glycemia 157 Glycoprotein 288 Glycosyltransferase 54 Gnawing 211

Goiter 30

GOT 75

Growth hormone 138

retardation 228 Guanylate cyclase 285 Guinea pig 262,323

-, stimulated ileum 215 Gum arabic 39

-, biological effects 47

- tragacanth 39

Half-life, phenytoin and

phenylbutazone 307 -, quinidine 301 Halogen substitution,

benzodiazepine 121 Haloperidol 210,216, 280 Hamster 262 Head bobbing 211 Healing of gastric

ulcers 37 Heart $39-$, guinea pig 316

- $\quad$ rate 17,21

Hemoglobin 187

Heparin 182

Hepatic antioxidants 341

ascorbate 341

biotransformation 163

blood flow 305

clearance of morphine 93

dysfunction 69

excretory function 69

glucose output 138

integrity 181

lipid peroxidation 341

metabolism 204, 307

microsomes 113

-, protein and cholesterol

concentrations 166

- $\quad$-, UDP-glucuronosyltransferase

activity 168

monooxygenase 201

-, inhibition by SKF 525-A 201 Hepatocytes 69, 182 Hepatoma, rat 246

Heroin addiction 227 Hexobarbital 47, 197 -, 14C 331

-, oxidative metabolism 89 Hexobarbitol 335

High speed liquid chromatography

98, 202 High spin, relationship in vivo and in

vitro 274 Hind paw 289 Histamine 32, Ill, 128, 259, 280,

323

H2-blockers 104

release 254

—, stress ulcer formation 36 Homovanillic acid 149 Hormones, thyroid 29 HSLC 98 Human alcoholemia 252 
- $\quad$ cultured lymphocytes 241

Huntington's chorea 210

Hyaluronidase 59

Hydratropic acid 323

Hydrocarbone, polycyclic aromatic

241 Hydrocortisone 56 /3-hydroxy-amphetamine 157 4-hydroxyantipyrine 99, 2014 hydroxycoumarin 233 6-hydroxydopa 8 6-hydroxydopamine 8 5-hydroxyindoleacetic acid 8 Hydroxylamine 116 Hydroxylase 163 -, benzo(a)pyrene 192 Hydroxylating systems 1983 hydroxymethylantipyrine 98, 201 5-hydroxytryptamine 8, 150, 254 Hyperglycemia 138, 143, 221 Hypertensive rats 316 Hypokinesia 149 Hypothalamus 1, 128, 133, 157 Hypothyroidism 21, 28

Ibuprofen 289

Implantation, morphine pellets 128

Index, accommodation 249

-, (3-blockade 303

Indocyanine green 69

Indomethacin 56, 289, 328

Inducibility, aryl hydrocarbon

hydroxylase 194 Induction, cytochrome P-450 and

cytochrome c reductase 341 Inflammation 50, 288

350

Subject Index

Infusion, 3-3H-glucose 138

-, quinidine 302

Inhibitory activity 292

Insulin 140, 158,221

$-, 14 \mathrm{C} 331$

Interindividual differences, plasma

decay of drugs 197 Iron, spin state of P-450 265 Irradiation, microwave 280 Ischemia, cerebral $15-$, myocardial 188 Isolated cat nictitating membrane

215

- $\quad$ perfused rat liver 69

Isoniazid 154

Isoproterenol 21, 221, 280, 301

Jaundice, cholestatic 69

Kidney 334 Kinins 188, 323

Lactate 181

Lactation 227

LDH activities, liver perfusate 184

Lesion formation, stomach 32

Leucine 86

Leukemia, murine LI210 61

Levodopa, effect of chronic

treatment 153 -, effect on vitamin B6 and 
dopamine metabolism 152 Lewis rats 289 Ligand field strength 262 Limbic system 210 Lip id dystrophy 158

mobilization 29

peroxidation 341

solubility 338 Lipids, unsaturated 163 Lipopolysaccharide 288 -, bacterial 182

Liquid chromatography 98

Litter size 228

Liver 39, 192, 334

-, cytochrome P-450 concentration

and NADPH cytochrome c

reductase activity $167-$, glucose output $161-$, human 241

-, isolated perfused 181 -, microsomes 241,262

mitochondria 39

monooxygenase 201 Locomotor activity 121,129

-, effects of desmethyldiazepam

and chlordesmethyldiazepam

122 Longitudinal smooth muscle, guinea

pig ileum 320 Long-term biological rhythms 88 Lorazepam 126 Low spin 274 Lung 334

-, adenylate cyclase 282 - , mouse 280 Lymphnode cells 288 Lymphocyte activation, inhibitors

288 Lymphocytes 292 -, mitogen-activated 191, 241 Lysosomal enzymes 297

- $\quad$ fragility and ultrastructure 181

Lysosomes 59

- , enlargement and vacuolization 181

Macaca mulatta 98, 201

Macroglobulins 288

Macrophages 192, 289

-, pulmonary 286

Man 323

Mannitol 338

-, 14C 331

MAO 1, 116

-, assay 3

-, type A and type B enzyme 1

Marihuana 79

-, anticholinergic activity 173

Mast cells 32, 36

- degranulation 254

-, stomach wall 254

Mean body weight under methadone

229 Measurements of calcium release and

uptake 316 Meclofenamate 328 Medulla 133 Membrane, lysosomal 181

- potential 249

- transport 330

Menstrual cycle 192

Meperidine 116

Mesolimbic system 212 
Metabolic activation 241

Metabolism, benzo(a)pyrene 191

-, carcinogen 197

-, hexobarbital 208 -, pyrine 192 Metabolite interference 98 Metabolites, antipyrine 98

Methacholine 32 Methadone, maintenance and detoxification programs 227

- treated rat mothers,

hyperactivity 230

Methionine enkephalin methylester

215 3-methylcholanthrene 192, 262 Methylcellulose $39 \alpha$-methyldopa hydrazine $211 \alpha$-methylpara-tyrosine, inhibitor of

catecholamine biosynthesis 212 Methylprednisolone, anti-

endoxotoxin actions 181 Metyrapone 264 Microcirculation 188 Microsomal calcium uptake 315

- enzymes 113

- $\quad$ monooxygenase 198

Microsomes, liver 163, 241

-, rat 207

Microwave irradiation 130, 280

Mitochondria, brain 1

- , hepatic 187

-, isolation 2

-, liver and heart 39

-, purification 2

Mixed function oxidase 39, 113

Monkey 98, 323

Monoamine oxidase, see MAO

Monocytes 192, 289

Monooxygenase activity 167

Monoprogargylamine 115

Morphine 88, 128, 215, 305

-, acute and chronic effects 128

-, brain and plasma levels 89

-, dependence 89

-, development of tolerance 128

-, effect oncAMP 134

Subject Index

351

-, hepatic clearance 89 -, pellet implantation 88 Mothers, drug-addicted 227 Motor activity 122

Mouse 262, 280

brain, adenyl cyclase activity 134

- , the level of free and total Ach

12

- $\quad$ vas deferens, electrically

stimulated 215

Mucosal hemorrhage 105

mast cell count 157 Muscle 334 
contraction 316 Mycobacterium butyricum 289

N, liquid 130 N-acetyl-p-aminophenol 192 NADPH cytochrome c reductase 163 Nalaxone 88, 216 Nalorphine 216 /3-naphtoflavone 262 Narcotic 227

- $\quad$ analgesics, a new model for the

study 215

N-benzyl-N-methyl-2-propynylamine,

MAO-inhibitor $113 \mathrm{~N}$-cyclopropyl-methyl-norazido-

morphine $215 \mathrm{~N}$-dealkylation, SKF 525-A $208 \mathrm{~N}$-demethylation, reduced rate in

pregnant rats $231 \mathrm{~N}$-desmethylantipyrine $98 \mathrm{~N}$-desmethyldiazepam $121 \mathrm{~N}$, N-dimethylformamide

271 6(N, N-diethylamino)hexyl 3,4,5-

trimethoxybenzoate $3158(\mathrm{~N}, \mathrm{~N}$-diethylamino)octyl 3,4,5-

trimethoxybenzoate

hydrochloride 320 Needle biopsy 203 Neuroleptics 69, 280 Neurological signs 211 Neurons,

glucosensitive 161 -, monoamine-containing 129 Neuro transmitters 128 -, false 213

Nerve accommodation 249 -, excitability properties 249 N-hexane 264

N-histidine 273

Nicergoline 15

Nicotine 339

$-, 14 \mathrm{C} 331$

Nictitating membrane, isolated

medial smooth muscle of the cat

215 p-nitroanisole 0-demethylase $163 \mathrm{~N}$-methylpropargylamine 115 Noradrenaline 1, 8, 83, 86, 128 ,

$139,150,216,315$-, dose-response curves of the aortic

strips 316 -, lipolytic action 29 Noradrenergic neurotransmission 215 Norepinephrine, see

noradrenaline Normophine 216 Norpargyline 113 Nutrition 227 Nystatin 288

Octylamine 262, 271

O-dealkylation, phenacetin 192

One-compartment, open model 308

Opiates 215

-, action 96

-, receptors 215,220

,- testing 219

Organ culture 50

Organic dye excretion 69

Ovarian cancer chemotherapy 330

Oxarbazole 323

Oxidative metabolism, hepatic 98

phosphorylation 39

-, uncoupling 187

Oxygen consumption $21,25,29,41$,

181,187 Oxymorphone 218 Oxyphenbutazone 56

P-450 262

-, differences in spin 274 -, ferric iron spin state 262 Pancreatectomy, reversible by

somatostatin 224 Pancreatic hormones 221 Papio anubis 222 
- $\quad$ cynocephalus 222

Pargyline, effects on hepatic

microsomes 114

Parietal cell 110

Parkinsonism 149, 210

-, reserpine-induced 210

Parturition 231

Patients, cancer 192

-, hypothyroid 29

PBI 27

Pellet implantation, morphine 135

Pentobarbital 182, 307, 335

-, 14C 331

Pentobarbitalphenole, I4C 331

Perfusion pressure, liver 181

Peritoneal cavity 330

- membrane 330

Peritoneum, buffering ability 332

Permeability 338

PGF2 $\alpha 323$

Phagocytic function, liver 187

Pharmacokinetics, quinidine 301

Phenacetin 191, 263, 307

—, plasma levels in smokers 192

$\varnothing$-phenethylamine 1

Phenformin 158

Phenobarbital 47, 341

-, hepatic microsomal enzymes and

lipid peroxidation 342 -, effect on levels of potential

antioxidants 342 -, induction of P-450 262 Phenothiazines 69, 116,285 Phenprocoumon 233

Phentolamine $30-$, plasma glucose, insulin and

growth hormone 145 Phenylbutazone 56, 289, 307, 323 -, pharmacokinetic parameters 311 -, serum protein binding 307 -, total and free serum levels 311 2-phenylethylamine, a putative neurotransmitter 210 Phenylpropanolamine 157 - blood glucose in diabetic rats 160 -, a potential antidiabetic 1625 -phenyltetrazole 57 Phenytoin 307

-, pharmacokinetic parameters 310 -, serum protein binding 307 -, total and free serum levels 310 Phosphatase, nonspecific 151 Phosphodiesterase 29, 128, 285

352

Subject Index

Phospholipids 163, 271

Physical dependence, morphine 88,

129 Phytohemaglutinin 288 Pimozide, a dopamine receptor

blocker 138 -, glucose, growth hormone and

cortisol 143 Piperonyl butoxide 264 Placenta 192 Plant exudates 45 Plasma elimination rates, phenacetin, 
acetanilide, theophylline,

antipyrine 195

glucagon 221

glucose 141

half-life, acetanilide 195

half-life, antipyrine 98, 202

insulin 138,141

protein binding, quinidine 303 Platelets 59

Polycyclic aromatic hydrocarbons,

metabolic activation 191 Polyethylene glycol, 14C 331 Polyoxyethylene (10)-nonylphenyl

ether 275 Polypropylene glycol, 14C 331 Pons 9

Porphyrin ring 274 Postischemic period 19 Postnatal body growth, retardation

231 Postpartum weight 228 Potassium conductance 249 -, contraction of the muscle 320

Pregnancy 227 Preparation, SRS-A 323 Procaine 320 Proline-3 H, incorporation in collagen

50 Propadrine 157 Propantheline bromide 104 Propranolol 138, 301, 325 -, effect on growth hormone 145 -, effect on quinitidine

pharmacokinetics 302 Propylthiouracil 21 Propynylamines 113,116 Prostaglandin 104 -, release 59

Protein binding 307

- $\quad$ catabolism 56

-, hepatic microsomal 163

kinase 285

synthesis 168, 297 Proteoglycan 50

-, incorporation of 3SS-sulphate 52

Proteolysis, hepatic 183

Proteolytic enzymes 56,169

Psychoactive drugs 280

Ptosis 211

Pulmonary cyclic nucleotides 280

- $\quad$ tissue, adenylate cyclase 280

Pump, microsomal calcium 321

Punishment 121

Pylorus-ligated rat, effects of THC

and tridihexethyl chloride 174 Pyridine 264 Pyridoxal kinase, determination 150

- phosphate, determination 151

Pyridoxine 149

QTc, prolongation 303 Quinidine, electrocardiographic

effects 301 Quinolinium dibromide 61 -, excretion 63 -, interaction with protein and

DNA $62-$, tissue distribution 64

Rabbit 262

—, acute renal failure 307

-, uraemic 307

Radioassay, antipyrine 98, 208

Rat, adjuvant arthritic 288

-, administration of phenobarbital 
341 -, aortic strips 316 -, arthritic 288 -, basal ganglia 149 -, fasted 79 -, gastric effect 254 -, hypoxic 20 -, gastrointestinal tract 79 -, hypothyroid 21

liver, levels of physiological antioxidants 341

-, perfused 68

-, nontolerant and tolerant 128

-, normotensive and hypertensive

315 -, pylorus-ligated 37, 173, 254 Reactive intermediates 241 Receptor blockade, histamine-H2 32 -, dopamine 139 -, muscarinic 176 -, opiate B 215 Release, histamine 32 Renal clearance, quinidine 301

failure 307 Renex 690275 Reserpine 86,210, 254

induced ulceration 254 Restraint immersion 104 Retardation, postnatal growth 228

Reticuloendothelial clearance

technique 182

- $\quad$ system 187

Retropulsion 211

$\alpha$-rhamnose 45

Rhesus monkey 98, 201

Rheumatoid arthritis 56

Rhythm, seasonal or circannual 88

Rigidity 149, 211

RNA 297

Salicylic acid 56, 339

-, 14C 331

-, incorporation of proline into

collagen 53 -, incorporation of sulphate into

proteoglycan 52 Salivation 91 Salsolinol 1

Sarcoplasmic reticulum 315 Scopolamine 110 Secobarbital 335 -, 14C 331

Second messenger, cAMP 128 Secretion, gastric acid 33 -, growth hormone 138 Serotonin 1, 83, 128, 150, 210, 323 -, uptake 86 Serotoninectomy 8 Serum albumin 233 -, arthritic 289

—, quinidine concentrations 304 Shay rat test 173 Shock 187

Subject Index

353

-, endotoxic 181

Side effects, anticholinergics 110

Sidman avoidance 121

SKF525-A 201

-, effect on aldrin epoxidation 206

-, effect on antipyrine 204

Skin 192

Sleeping time, pentobarbital 165

Slow-reacting substance of

anaphylaxis 323 Smoking, marihuana 173 Smooth muscle 315 Sniffing 211 Sodium bicarbonate, effect on gastric

ulcers 258 
- $\quad$ conductance 249

Solvent systems, extraction

efficiency 100 Somatostatin 104, 221 Spasmogens 326 Spectrophotometric methods 98

Spectrophotometry, off-balance 262 Spin state, P-450 iron in vivo 262,

268 Spleen 289 SRS-A 323

Stabilization of membranes 186 Steroid hormones 231 Stereotypy 210

Stomach, glandular mucosa 35,254 -, perfused in situ 32

- wall, examination of mast cells

255

Strangulation 19 Streptozocin 157 Stress 36

- $\quad$ ulcer formation 259

Striatum 8, 129

Strips, aortic 316

Studies, electroencephalographic

123 Substance P 210

Substances, immunosuppressive 295 Sucrose 338 Sulfobromophthalein, biliary

clearance and transport

maximum 69 Sulfonylurea 158 Sulphate, incorporation in

proteoglycans 50

Supra-additive effects 106 Sympathectomy, chemical 13 Synaptic elements, cAMP 128

Synaptosomal uptake, tryptophan

and choline 83 Synaptosomes 83 Systolic blood pressure 21

Tachycardia 19, 303 Tail-flick assay 130 Taurocholate 74 Taurolithocholate 76 Temperature, colonic 27 -, tail skin 21

Teratogenicity, methadone 227 Testosterone 88

Tetrahydrocannabinol $79,83,173-$, uptake of tryptophan and

choline 83 Tetrahydrofuran 264 Tetrahydroisoquinoline 2, 155 Tetrahydropapaveroline 1

Tetrazoles, substituted 56 Theophylline 21, 191 Thickening agents 39 Thiolate 274 Thiopental

307, 335 -, 14C 331 Thioxanthene 69 Thromboxane 328 Thymidine, 3H 288 Thyroidal uptake of

1MI 27 Thyroid gland 21 Thyroxin 21 Tissue slices of mouse lung, effect of

noradrenaline on cAMP 282 Tolbutamide 158, 307 Tolerance 89, 128 Toluene 263

Total clearance, quinidine 301 Tracheal chain, guinea pig 323 - pressure 325 Transcellular

permeability 338 Transport, model compounds 330 -, peritoneal 330 Treatment, ulcer 173

Tremor 149, 211 Tremorine 173 Tricyclic antidepressants 116 Tridihexethyl chloride 173

Trypsin 56, 163

Tryptamine 1

Tryptophan 83

Tuberculostatic drug, anti-vitamin B6

effect $154 \mathrm{~T}$-wave amplitude 303 Tyramine 1 Tyrosine 150

- decarboxylase 151

UDP-glucuronosyltransferease 163

Ulcer, formation 32, 173, 179, 254

- , gastric and duodenal 104

Uptake, 131I 21

-, thymidine 295

-, tryptophan and choline 83 
Uranyl nitrate 307

Urea, 14C 331

Urethane 47

Urination 91

Urinary excretion,

2-phenylethylamine 210

- recovery, quinidine 303

Vagal overactivity 260

- $\quad$ stimulation 32

Vascular smooth muscle 316

Vasodilation 187

Verapamil 316

Visual disturbances 158 Vitamin B6 149 Voltage clamp 249

Warfarin 233, 307 Water consumption, under methadone 229

insoluble compounds 39

intake 25

Wet dog shakes 88 Withdrawal 88, 227

Xenobiotic, membrane transport 330

- $\quad$ substrates 93

$\alpha$-xylose 45

Xylosyltransferase 54

Zinc 32

-, anti-ulcer action 36,254

Zoxazolamine 197 